\title{
Detrusor Underactivity and Underactive Bladder in Women: What Is New?
}

\author{
N. I. Osman ${ }^{1} \cdot$ K. H. Pang ${ }^{2} \cdot$ F. Martens ${ }^{3} \cdot$ T. Atunes-Lopes ${ }^{4} \cdot$ B. Geavlete ${ }^{5} \cdot$ T. Husch ${ }^{6} \cdot$ M. Tutolo ${ }^{7} \cdot$ M. S. Rahnama'i ${ }^{8}$. \\ T. Marcelissen ${ }^{9} \cdot$ EAU Young Academic Urologists (YAU) Functional Urology Working Group
}

Published online: 27 November 2019

(C) The Author(s) 2019

\begin{abstract}
Purpose of Review Detrusor underactivity (DU) and its symptom-based correlation, the underactive bladder (UAB), are common problems encountered in urological practice. Whilst DU has been defined for many years, only recently has UAB received a formal definition and there is now accumulating literature based on this condition. In this article, we reviewed the recent literature on the aetiology, pathogenesis, diagnosis and management of DU/UAB in women.

Recent Findings Detrusor underactivity is diagnosed on urodynamic studies but there still remains a lack of widely recognised and accepted diagnostic criteria. Commencing treatment based on the diagnosis of UAB, with or without a PVR measurement, is perhaps feasible due to the lower occurrence of BOO in women and specific clinical features that may distinguish this group. Prospective studies attempting to correlate UAB with the underlying DU are needed before this approach could be considered. Summary Detrusor underactivity/UAB in women requires a separate consideration from men due to the anatomical and functional differences in the lower urinary tracts between both genders and consequent differences in pathologies affecting them. The aetiology of DU remains largely unknown, but is probably multifactorial, including myogenic, neurogenic and vasculogenic factors. There remains a lack of any simple effective drug treatments, whilst apart from sacral neuromodulation for the specific subgroup with non-obstructive urinary retention, no safe and effective surgical treatment is currently available. Often, permanent or intermittent bladder drainage with a catheter is the final solution.
\end{abstract}

Keywords Detrusor underactivity · Acontractile detrusor · Underactive bladder

\section{Introduction}

The problem with voiding difficulty due to insufficient bladder contraction has received a great deal of attention

This article is part of the Topical Collection on Voiding Dysfunction Evaluation

N. I. Osman

nadirosman@gmail.com

K. H. Pang

karlpang@doctors.org.uk

F. Martens

frank.martens@ radboudumc.nl

T. Atunes-Lopes

tiagoantuneslopes@gmail.com

B. Geavlete

bogdan_geavlete@yahoo.com in recent times [1, 2]. Detrusor underactivity (DU) refers to the urodynamic finding of impaired bladder contraction, whereas the underactive bladder (UAB) is defined as the collection of associated symptoms. An UAB leads

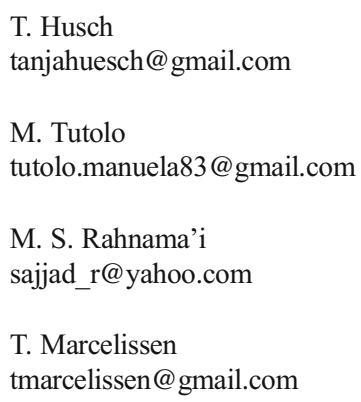

Extended author information available on the last page of the article 
to incomplete emptying of the bladder, it can impact quality of life and result in complications such as urinary tract infections (UTI). It has been acknowledged that the scale of the problem may have been previously underestimated and there has been a recent expansion in both clinical and scientific research concerning UAB aetiology, investigation and pathophysiological mechanisms [3]. Whilst sharing some common factors, the aetio-pathogenic basis of DU/UAB in women is distinct from that in men due to the functional and anatomical differences seen in the male and female urinary tracts. These differences also have implications on how the problem is assessed and managed. In this article, we shall focus on the recent literature and discuss what is already known on the aetiology, pathogenesis, diagnosis and management of DU/UAB in women.

\section{Terminology and Definitions}

Although there is a plethora of terms that refer to impaired voiding function, the term adopted by the international continence society (ICS) in the 2002 standardisation document is DU. This is defined as "a contraction of reduced strength and/or duration, resulting in prolonged bladder emptying and/or failure to achieve complete bladder emptying within a normal time span" [4]. Despite the fact that this definition does not provide specific parameters for strength and length of contraction, bladder emptying and voiding time, it provides a good framework for considering which aspects of voiding may be affected. These parameters are poorly defined in healthy men and women and are likely to vary with age. The term UAB is much more recent and has been considered to include symptoms associated with DU, similar to the definitions and association between overactive bladder (OAB) and detrusor overactivity (DOA). Recently, an international consensus group developed a definition for UAB on behalf of the ICS, stating that "UAB is characterized by a slow urinary stream, hesitancy, and straining to void, with or without a feeling of incomplete bladder emptying sometimes with storage symptoms" [5•]. Thus, UAB, like OAB, is a "symptom complex." From a semantic point of view, it is important to distinguish the term symptom complex from the term symptom syndrome. A syndrome refers to a group of symptoms and clinical signs which relate to the deviation of the function of an organ or a lesion in the organ. A symptom complex is a group of symptoms or clinical signs without a lesion or functional disturbance of a particular organ. The underactive bladder is characterised by a group of symptoms which may or may not be associated with urodynamic abnormalities.

\section{Epidemiology}

Understanding the prevalence of UAB from a population perspective was previously not possible due to the lack of an agreed definition. Even currently, it is not yet clear how many patients whose symptoms fit the new definition of UAB actually have an underlying urodynamic dysfunction. Therefore, at present, gathering epidemiological data using internet surveys, etc., as in some of the large studies into $\mathrm{OAB}$, is perhaps premature. However, traditionally speaking, bladder outlet obstruction (BOO) in symptomatic women is considered quite rare and so the symptoms of $\mathrm{UAB}$ are much more likely to correlate with underlying DU in women as compared with men. The available prevalence data largely resides from clinical studies of patients with lower urinary tract symptoms (LUTS) assessed using urodynamic studies in secondary care. This has shown DU to be present in $12-45 \%$ of females with LUTS, distinctively more common in elderly nursing home residents who often have concomitant DOA, an entity described as "detrusor hyperactivity with impaired contractility" (DHIC) [1]. A recent study from China which analysed 3265 urodynamic traces of neurologically normal women, using the ICS definition of DU, found a prevalence of $19.9 \%$ [6]. Another recent large series from Taiwan including a total of 1914 women found a DU prevalence of $17.6 \%$ and detrusor hyperactivity with impaired contractility of $12.1 \%$, the diagnosis was made on the basis of videourodynamics with no specific definition added [7]. Valentini and co-workers specifically looked at women over the age of 80 and found that of 169 consecutive LUTS patients, DU was present in $38.8 \%$ of the cases consistent with other data, thus suggesting the problem to become more frequent with advancing age [8]. The major issue with such clinical studies assessing urodynamic data are the heterogenous patient groups, the variation in the definition of DU used and diagnostic criteria.

Very little population data is available due the previously mentioned difficulties. A recent cross-sectional survey of 291 women in North America showed symptoms of difficulty emptying the bladder to be present in $20 \%$ of women. Another recent study from North America looked specifically at rates of urinary retention and catheter use in women receiving health care through Medicare [9]. A 5\% sample of Medicare claims was assessed, as the authors applied comparative methodology (using age-matched controls who did not have retention or had not undergone catheterisation). The authors estimated the retention rates to be 1532 per 100,000 Medicare beneficiaries in 2012, with 108 per 100,000 requiring chronic catheterisation. Multivariable regression showed that UTI, pelvic organ prolapse and neurological disease were positively associated with retention and catheterisation necessity. In a new report from Finland, Nuotio and colleagues assessed the factors associated with elevated post-void residual (PVR) and its prognostic significance in older women who 
had hip fractures [10]. When analysing 409 women aged $>65$, they found a raised PVR (defined as $160 \mathrm{ml}$ or more) in 15.6\% of the cases. Having urinary or faecal incontinence, difficulties in physical activities of daily living, malnutrition, poor performance on Timed Up and Go and Elderly Mobility Scale were all significantly associated with an elevated PVR. In addition, having elevated PVR was significantly associated with an increased risk of mortality at 1 year. The authors suggested that PVR should be included in the comprehensive geriatric assessment.

\section{Aetiology}

The aetiological factors in women largely mirror those found in men. Aetiological factors can be broadly grouped as neurogenic, myogenic whilst more recently, vasculogenic aetiologies are increasingly considered. Neurogenic aetiologies can be considered affecting sensory nerves, motor nerves or central brain circuits and include problems such as stroke, multiple sclerosis, Parkinson's disease and traumatic injury. Myogenic aetiologies are less definable but still BOO has certainly been shown to result in bladder wall fibrosis according to multiple animal experiments. However, BOO in women is uncommon, the causes of which include the sequelae of incontinence surgery (particularly mid urethral slings), pelvic organ prolapse and urethral stricture.

The influence of pelvic arterial ischaemia on bladder function is generating increasing interest [11]. Experiments in rodents have shown that inducing ischaemia in the pelvic vasculature leads to ischaemia in the bladder wall and impaired oxygenation. The outcome is consequently represented by oxidative stress, ultrastructural changes consistent with tissue injury and denervation. Clinically, animals will initially show DOA and features of OAB before progressing to DU. It has been hypothesised that in man, DU is not a separate entity to DOA but is merely the end stage of untreated DOA [12]. This may explain the increase in DU in older groups. Recent work in female rats, comparing older and younger groups, have also suggested that autonomic dysfunction at various levels may also be contributory [13].

Whether urodynamic variables are related to certain aetiologies of UAB in women is a question that was recently explored by Brown and co-workers [14•]. The authors retrospectively reviewed data on 200 women acquired over 4 years at a single institution. Presumed aetiologies were grouped as cardiovascular disease manifestation, cardiac risk factors, neurological or idiopathic. There were no differences in urodynamic parameters between groups. Diabetic bladder dysfunction is a recognised cause of DU/UAB. A recent study by Malik et al. prospectively assessed symptoms and urodynamic parameters in 384 women of whom $26 \%$ were diabetic and revealed no differences in presenting symptoms between the two groups.
However, as expected, diabetic patients were more likely to have abnormal urodynamic features. Specifically, the duration of diabetes longer than 10 years was associated with lower detrusor pressure during voiding and higher PVR [15].

\section{Diagnosis}

The underactive bladder is described as a "symptom complex." In women, due to a lower occurrence of $\mathrm{BOO}$ when compared with men, it is likely that UAB is more closely correlated to underlying DU on urodynamic studies. Bladder outlet obstruction, although considered uncommon, has recently been found in as many as $10 \%$ of women with LUTS [16] and in up to one-third of patients in older series [17•]. It would therefore be potentially useful to identify whether any specific clinical variables are more closely associated with DU as compared with BOO. In a relatively large study (1788 patients) assessing symptoms and bladder diary variables in patients with DU and other urodynamic dysfunctions, Gammie and colleagues found no significant differences with regard to any of the variables in the DU and BOO groups [18••]. However, patients with DU were more likely to report stress urinary incontinence (SUI, OR 1.91), reduced stream (OR 1.81 ), straining to void (OR 3.13), enuresis (OR 1.98), absent or reduced sensation (OR 8.56), previous urinary retention (OR 2.94) and use of antidepressant (OR 4.34).

Detrusor underactivity is diagnosed on urodynamic studies but there still remains a lack of widely recognised and accepted diagnostic criteria. Several common approaches to establishing the diagnosis have been taken, most of these methods assessing detrusor contraction strength, although this is only one aspect of efficient voiding. Other aspects include contraction speed and length.

(1) Cut offs for urodynamic variables, e.g., detrusor pressure at maximal flow (pdet@ Qmax), maximal flow (Qmax), with or without other parameters, e.g., PVR. However, the normal ranges are not agreed upon and moreover, limited as a low Qmax could also be due to BOO, whilst a low pdet@Qmax might be related to reduced outlet resistance, in the case of women with SUI. Moreover, it is possible to have DU and normal or low PVR, as recently demonstrated by Khayyami and colleagues in a small retrospective study on 20 women with voiding dysfunction [19].

(2) Calculations that estimate the isovolumetric pressure, e.g., projected isovolumetric pressure (PIP) [20] or bladder contractility index (BCI). This approach is the simplest to use in clinical practice. Both PIP and BCI are based on the same calculationPdet@Qmax+KQmax, where $K$ is a fixed constant representing the slope of the bladder outlet relation. The $\mathrm{K}$ value is dependent on the studied population (sex and age group). In men with $\mathrm{BPH}$, it is taken as $5 \mathrm{cmH}_{2} \mathrm{O} / \mathrm{ml} / \mathrm{s}$ whilst in older women $1 \mathrm{cmH}_{2} \mathrm{O} / \mathrm{ml} / \mathrm{s}$ was found to be more 
accurate [21]. This is an important point to note to avoid mistakenly applying the wrong $K$ value in female patients.

(3) Urodynamic stop tests interrupt the flow to measure isovolumetric pressures [22]. There are two types of stop test, voluntary or mechanical (using a catheter balloon to occlude the urethral lumen). The voluntary stop requires an intact sphincter mechanism, hence may be difficult or impossible to perform in some women. The mechanical is better in this regard; however, it does not allow the simultaneous measurement of flow. Additionally, it can be painful; hence, is generally not performed in clinical practice.

It is not uncommon for patients to fail to void during standard urodynamics due to an element of anxiety. This phenomenon has been termed the "bashful bladder." The postulated cause is an inability to appropriately relax the pelvic floor which results in reflex inhibition of the detrusor. A careful history is usually taken to distinguish a bashful bladder from DU. A more accurate method may be to perform ambulatory urodynamics. Van Koevering et al. showed that $84 \%$ of patients who failed to generate a detrusor contraction during standard urodynamics had a demonstrable contraction during an ambulatory study [23•].

\section{Management}

At present, there is no widely available drug or surgical treatment for UAB/DU. This lack of a simple and effective treatment has often led clinicians to view UAB/DU with a somewhat nihilistic viewpoint. The mainstay of management has been to try and reduce the burden of UAB symptoms (and additionally those exacerbated by DU such as SUI) and reduce the chance of complications associated with incomplete bladder emptying such as UTI, bladder stones and upper tract deterioration. The management approaches can be categorised as those that aim to enhance bladder contraction, those that attempt to reduce outlet resistance and those that bypass the problem. The choice of management strategy depends on multiple factors including aetiology, age, sex, cognition and dexterity, patient choice, surgeon experience, surgical volume and cost considerations.

\section{Conservative Approaches}

In women who void infrequently as a consequent of sensory impairment or a central nervous system problem, timed voiding may be trialled and may have some success in reducing leakage associated with a full bladder or UTI. Double voiding aimed to facilitate a more complete bladder emptying should be advised as an initial measure and often helps reduce the PVR. Bladder compression (crede's manouver) is generally avoided, as it can generate high intravesical pressure and reflux, apart from the specific situation in which the urinary sphincter mechanism is known to be incompetent.

Pelvic floor physiotherapy is an option in women who are considered to have dysfunctional voiding [24]. Pelvic floor contraction in these patients leads to an inhibitory effect on detrusor contraction. The treatment is usually given with biofeedback techniques. Van Koeveringe and colleagues showed that $24 \%$ of patients with acontractile detrusors on standard pressure flow studies but some evidence of preserved contractility according to ambulatory studies could be successfully treated with physiotherapy [23•].

In cases in which the issue is elevated PVR, a decision should be made whether to institute clean intermittent selfcatheterisation (CISC). It is not always necessary for this to be done even if the PVR is large. Provided that patients' symptoms are tolerable and there is no evidence of complications, a safe approach is to monitor the cases by ultrasound and serum biochemistry. Due to the lower pressure outlet in ageing women, the chance of upper tract compromise is decreased compared with men. Alternative forms of catheterisation if CISC is not possible are constituted by an indwelling urethral catheter, which is not advised in women due to the risk of urethra atrophy and worsening incontinence, or indwelling suprapubic catheter which is preferable. In either scenario, intermittent drainage should be practiced when possible in order to maintain bladder capacity [25].

\section{Pharmacotherapy}

Presently, there is no effective drug treatment for women with $\mathrm{UAB} / \mathrm{DU}$. Several agents have been studied in various contexts. The most widely studied agents are the parasympathomimetics. Barendrecht et al. systematically reviewed the literature on their use (different agents studied) and found a heterogenous group of studies with different populations and various settings (e.g., postoperative, postpartum). Only four out of the ten evaluated randomised studies showed a significant advantage over placebo; thus, the review concluding that there is little if any benefit [26]. The only study in women involved over 30 years old patients with elevated PVR and showed no advantage of bethanachol chloride over placebo [27]. In a recent study, Gaitonde and colleagues looked at the National ambulatory care survey in the USA in the decade 2003 to 2013 [28]. A sample of 17,321,630 patient visits of women $>18$ years with LUTS or urinary retention was assessed. In total, $0.8 \%$ of visits included a prescription of bethanachol. Typically, it was prescribed in elderly women (mean age 62.3 years) on an ongoing basis $(79 \%)$ mostly by urologists (92\%) for atony of bladder (35\%), urinary retention $(20 \%)$, neurogenic bladder $(18 \%)$, urinary incontinence $(16 \%)$ and incomplete bladder emptying (10\%). Thus, bethanachol continues to be prescribed despite a lack of convincing evidence. It must be remembered that parasympathomimetics are 
associated with significant side effects which are dose dependent (e.g., bronchospasm, abdominal cramp, nausea and flushing) and rarely severe cardiac depression can occur leading to cardiac arrest.

\section{Surgical Options}

Sacral neuromodulation is the most well-studied surgical approach in women with DU, the specific subgroup with nonobstructive urinary retention (or Fowler's syndrome) [29] and has well-established efficacy. Urethral dilatation continues to be used by some practitioners in women with DU and elevated PVR; however, an evidence base for such a practice is lacking. Others have advocated transurethral incision of the bladder neck (TUI-BN) in women with DU. In a recent study of 50 women with DU who did not improve after medical treatment, Jhang and co-workers performed TUI-BN [30] using two incisions at 5 and 7 o'clock. Patients were followed for a mean period of 61.8 months. The mean voiding efficiency increased from 0 to $50 \%(p<0.001)$ whilst PVR declined from 400 to $150 \mathrm{ml}(p<0.001)$. In total, approximately half of the patients were described as having a satisfactory outcome (voiding efficiency of $>50 \%$ ). However, following surgery, $16 \%$ of the cases described nocturnal enuresis and 6\% developed SUI, all of whom were said to have recovered continence by 6 months post-op. Only one patient developed a vesicovaginal fistula. Clearly, firm conclusions cannot be based on small retrospective studies such as this and the incidence of complications associated with this procedure is concerning. Reconstructive surgery to re-establish bladder contraction by way of the detrusor myoplasty was previously reported, both the rectus muscle and the lattismus dorsi have been utilised. Long-term outcomes have shown a resumption of spontaneous voiding in a majority or patients (17/24); however, one-third of patients who had complications include venous thrombosis, pelvic abscess and wound infection [31]. An alternative procedure, reduction cystoplasty has also been reported in historical series [32]. Both approaches are considered experimental in contemporary practice.

\section{Other Considerations}

The presence of DU may impact upon the management of other lower urinary tract dysfunctions, in particular the management of SUI. Natale and co-workers recently investigated whether DU is a risk factor for voiding dysfunction after transobutrator tape insertion in one of few such studies [33]. A total of 50 patients with normal contractility (PIP 30-75) and 50 patients with reduced contractility were prospectively studied. Voiding dysfunction was considered present based on questionnaire assessment. There was no significant difference in outcome with regard to continence between the two groups, but de novo voiding dysfunction occurred in $2 \%$ of the normal contractility group by comparison with $36 \%$ of the reduced contractility group. The authors concluded that preoperative urodynamics are useful in order to better counsel patients as to the risk of postoperative voiding problems. Ko et al. recently assessed the outcome of readjustable Remmeex suburethral sling in patients with SUI and DU, whereby the sling tension is adjusted on the first postoperative day based on stress test and uroflowmetry [34]. A total of 27 patients were followed for a mean period of 38 months' postoperatively. The treatment success in terms of continence was $81.5 \%$, whilst however, $25.9 \%$ of the cases requested sling removal due to persistent urinary retention during the first year. There was a significant decrease in flow rate (from 12.6 to $8.9 \mathrm{ml} / \mathrm{s}, p=$ 0.044 ) but no change in PVR. Clearly, larger studies are needed before any firm conclusion on this approach can be drawn. In any case, the high number of patients requesting sling removal for urinary retention needs to be considered.

\section{Conclusions}

In this article, we have discussed the most recent evidence pertaining to the problem of DU/UAB in women. Clearly, DU is a common bladder dysfunction in women and recent studies have suggested that it is more common with ageing. The UAB has only been recently defined and there is a clear need for some epidemiological studies whilst aiming to establish the prevalence among the general population. Commencing treatment based on the diagnosis of UAB, with or without a PVR measurement, is perhaps feasible due to the lower occurrence of $\mathrm{BOO}$ in women and specific clinical features that may distinguish this group. Prospective studies attempting to correlate $\mathrm{UAB}$ with the underlying DU are needed before this approach could be considered. Our understanding of the potential pathophysiologies at play is growing and in particular the possibility of the multifactorial nature of the issue in an individual patient. However, there remains a lack of any simple effective drug treatments, whilst apart from sacral neuromodulation for the specific subgroup with nonobstructive urinary retention, no safe and effective surgical treatment is currently available. The impact of DU on the treatment of other conditions such as SUI needs to be further explored whilst aiming to better understand which patients are at risk of developing postoperative voiding problems.

\section{Compliance with Ethical Standards}

Conflict of Interest Bogdan Geavlete has received speaker fees from General Electric Healthcare, Olympus and Ipsen. Nadir Osman has received speaker fees and travel grant from Astellas. Sajjad Rahnama'i has received speaker fees for Janssen and Bioness and a travel grant from Astellas. Karl Pang, Frank Martens, Tiago Antunes-Lopes, Tanja Huesch and Tom Marcelissen declare that they have no conflict of interest. 
Human and Animal Rights and Informed Consent This article does not contain any studies with human or animal subjects performed by any of the authors.

Open Access This article is distributed under the terms of the Creative Commons Attribution 4.0 International License (http:// creativecommons.org/licenses/by/4.0/), which permits unrestricted use, distribution, and reproduction in any medium, provided you give appropriate credit to the original author(s) and the source, provide a link to the Creative Commons license, and indicate if changes were made.

\section{References}

Papers of particular interest, published recently, have been highlighted as:

- Of importance

•- Of major importance

1. Osman NI, Chapple CR, Abrams P, Dmochowski R, Haab F, Nitti $\mathrm{V}$, et al. Detrusor underactivity and the underactive bladder: a new clinical entity? A review of current terminology, definitions, epidemiology, aetiology, and diagnosis. Eur Urol. 2014 Feb;65(2):38998.

2. Osman NI, Esperto F, Chapple CR. Detrusor underactivity and the underactive bladder: a systematic review of preclinical and clinical studies. Eur Urol. 2018 Nov;74(5):633-43.

3. Osman NI, Chapple CR. Contemporary concepts in the aetiopathogenesis of detrusor underactivity. Nat Rev Urol. 2014 21;11(11):639-48.

4. Abrams P, Cardozo L, Fall M, Griffiths D, Rosier P, Ulmsten U, et al. The standardisation of terminology of lower urinary tract function: report from the Standardisation Sub-committee of the International Continence Society. Neurourol Urodyn. 2002;21(2): 167-78.

5. Chapple CR, Osman NI, Birder L, Dmochowski R, Drake MJ, van Koeveringe $\mathrm{G}$, et al. Terminology report from the International Continence Society (ICS) Working Group on Underactive Bladder (UAB). Neurourol Urodyn. 2018 Nov;37(8):2928-31. First terminology document on the definition of underactive bladder.

6. Wang L, Wang C, Qu C, Yin L, Xu D, Cui X, et al. Relationship between urodynamic patterns and lower urinary tract symptoms in Chinese women with a non-neurogenic bladder. Asian J Urol. 2016 Jan;3(1):10-9.

7. Hsiao S-M, Lin H-H, Kuo H-C. Videourodynamic studies of women with voiding dysfunction. Sci Rep. 2017 Dec 28;7(1):6845.

8. Valentini FA, Marti BG, Robain G. Do urodynamics provide a better understanding of voiding disorders in women over 80 ? Prog Urol. 2018 Mar;28(4):230-5.

9. Cohn JA, Ni S, Kaufman MR, Graves AJ, Penson DF, Dmochowski RR, et al. Urinary retention and catheter use among U.S. female Medicare beneficiaries: prevalence and risk factors. Neurourol Urodyn. 2017 Nov;36(8):2101-8.

10. Nuotio MS, Luukkaala T, Tammela T. Elevated post-void residual volume in a geriatric post-hip fracture assessment in womenassociated factors and risk of mortality. Aging Clin Exp Res. 2019 Jan 9;31(1):75-83.

11. Vale L, Jesus F, Marcelissen T, Rieken M, Geavlete B, Rahnama'i MS, et al. Pathophysiological mechanisms in detrusor underactivity: novel experimental findings. Low Urin Tract Symptoms. 2019 Mar;12.
12. Chancellor MB. The overactive bladder progression to underactive bladder hypothesis. Int Urol Nephrol. 2014 Sep 20;46(S1):23-7.

13. de Oliveira MG, Alexandre EC, Bonilla-Becerra SM, Bertollotto GM, Justo AFO, Mónica FZ, et al. Autonomic dysregulation at multiple sites is implicated in age-associated underactive bladder in female mice. Neurourol Urodyn. 2019 Apr 1;nau.23990.

14. Brown ET, Cohn JA, Kaufman MR, Dmochowski RR, Reynolds WS. Phenotyping women with detrusor underactivity by presumed etiology: is it plausible? Neurourol Urodyn. 2017 Apr;36(4):11514. This study explored possible phenotyping for underactive bladder and the possibility of personalised treatment.

15. Malik RD, Cohn JA, Volsky J, Garvey S, Chang C, Bales GT, et al. A modern comparison of urodynamic findings in diabetic versus nondiabetic women. Female Pelvic Med Reconstr Surg. 2018 Apr;20:1.

16. Nitti VW, Tu LM, Gitlin J. Diagnosing bladder outlet obstruction in women. J Urol. 1999 May;161(5):1535-40.

17. Jeong SJ, Kim HJ, Lee YJ, Lee JK, Lee BK, Choo YM, et al. Prevalence and clinical features of detrusor underactivity among elderly with lower urinary tract symptoms: a comparison between men and women. Korean J Urol. 2012 May;53(5):342. Differences in underactive bladder between men and women are highlighted in this study.

18.• Gammie A, Kaper M, Dorrepaal C, Kos T, Abrams P. Signs and symptoms of detrusor underactivity: an analysis of clinical presentation and urodynamic tests from a large group of patients undergoing pressure flow studies. Eur Urol. 2016 Feb;69(2):361-9. In this study, urodynamic and clinical variables that are associated with underactive bladder are evaluated.

19. Khayyami Y, Klarskov N, Lose G. Post-void residual urine under $150 \mathrm{ml}$ does not exclude voiding dysfunction in women. Int Urogynecol J. 2016 Mar 30;27(3):467-73.

20. Schäfer W. Analysis of bladder-outlet function with the linearized passive urethral resistance relation, linPURR, and a disease-specific approach for grading obstruction: from complex to simple. World $\mathrm{J}$ Urol. 1995;13(1):47-58.

21. Griffiths D. Detrusor contractility-order out of chaos. Scand J Urol Nephrol Suppl. 2004 Jan 9;38(215):93-100.

22. Sullivan M, Yella S. Functional studies to assess bladder contractility. J urol Urogynakol. 2007;14(1):7-10.

23. van Koeveringe GA, Rahnama'i MS, Berghmans BCM. The additional value of ambulatory urodynamic measurements compared with conventional urodynamic measurements. BJU Int. 2010 Feb;105(4):508-13. This study showed that ambulatory urodynamics can provide a more accurate diagnosis of detrusor underactivity than conventional urodynamics.

24. Minardi D, d'Anzeo G, Parri G, Polito M, Piergallina M, El Asmar $Z$, et al. The role of uroflowmetry biofeedback and biofeedback training of the pelvic floor muscles in the treatment of recurrent urinary tract infections in women with dysfunctional voiding: a randomized controlled prospective study. Urology. 2010 Jun;75(6):1299-304.

25. Kristiansen P, Pompeius R, Wadström LB. Long-term urethral catheter drainage and bladder capacity. Neurourol Urodyn. 1983;2(2): 135-43.

26. Barendrecht MM, Oelke M, Laguna MP, Michel MC. Is the use of parasympathomimetics for treating an underactive urinary bladder evidence-based? BJU Int. 2007 Apr;99(4):749-52.

27. Barrett DM. The effect of oral bethanechol chloride on voiding in female patients with excessive residual urine: a randomized doubleblind study. J Urol. 1981 Nov;126(5):640-2.

28. Gaitonde S, Malik RD, Christie AL, Zimmern PE. Bethanechol: is it still being prescribed for bladder dysfunction in women? Int $\mathrm{J}$ Clin Pract. 2018 Aug; 15:e13248.

29. Tutolo M, Ammirati E, Heesakkers J, Kessler TM, Peters KM, Rashid T, et al. Efficacy and safety of sacral and percutaneous tibial 
neuromodulation in non-neurogenic lower urinary tract dysfunction and chronic pelvic pain: a systematic review of the literature. Eur Urol. 2018 Mar 11;73(3):406-18.

30. Jhang J-F, Jiang Y-H, Lee C-L, Kuo H-C. Long-term follow up and predictive factors for successful outcome of transurethral incision of the bladder neck in women with detrusor underactivity. J Formos Med Assoc. 2016 Sep;115(9):807-13.

31. Gakis G, Ninkovic M, van Koeveringe GA, Raina S, Sturtz G, Rahnama'i MS, et al. Functional detrusor myoplasty for bladder acontractility: long-term results. J Urol. $2011 \mathrm{Feb}$;185(2):593-9.

32. Klarskov P, Holm-Bentzen M, Larsen S, Gerstenberg T, Hald T. Partial cystectomy for the myogenic decompensated bladder with excessive residual urine. Urodynamics, histology and 2-13 years follow-up. Scand J Urol Nephrol. 1988;22(4):251-6.
33. Natale F, Illiano E, Zucchi A, Balzarro M, La Penna C, Costantini E. Transobturator mid-urethral sling in females with stress urinary incontinence and detrusor underactivity: effect on voiding phase. Int Urogynecol J. 2019 Feb;4.

34. Ko KJ, Suh YS, Sung HH, Ryu GH, Lee M, Lee K-S. Assessing the readjustable sling procedure (Remeex system) for female stress urinary incontinence with detrusor underactivity. Int Neurourol J. 2017 Jun 30;21(2):116-20.

Publisher's Note Springer Nature remains neutral with regard to jurisdictional claims in published maps and institutional affiliations.

\section{Affiliations}

\section{N. I. Osman ${ }^{1} \cdot$ K. H. Pang ${ }^{2} \cdot$ F. Martens ${ }^{3} \cdot$ T. Atunes-Lopes $^{4} \cdot$ B. Geavlete ${ }^{5} \cdot$ T. Husch $^{6} \cdot$ M. Tutolo $^{7} \cdot$ M. S. Rahnama'i ${ }^{8}$. T. Marcelissen ${ }^{9}$ E EAU Young Academic Urologists (YAU) Functional Urology Working Group}

1 Department Reconstructive Urology, Royal Hallamshire Hospital, Sheffield, UK

2 Academic Urology Unit, University of Sheffield, Sheffield, UK

3 Department of Urology, Radboud University Medical Center, Nijmegen, The Netherlands

4 Department of Urology, Hospital S. João, Porto, Portugal

5 Department of Urology, Saint John Emergency Clinical Hospital, Bucharest, Romania
6 Department of Urology, Universitätsmedizin der Johannes Gutenberg-Universität, Mainz, Germany

7 Department of Urology, URI, IRCCS Ospedale San Raffaele, Milan, Italy

8 Department of Urology, Uniklinik Aachen RWTH, Aachen, Germany

9 Department of Urology, Maastricht University Medical Centre, Maastricht, The Netherlands 